\title{
ORIGINAL
}

\section{RELACIÓN EN ESPÃ̃A ENTRE LA INVESTIGACIÓN SANITARIA FINANCIADA POR EL SISTEMA NACIONAL DE SALUD Y LA CARGA DE ENFERMEDAD EN LA COMUNIDAD}

\author{
Ferrán Catalá López $(1,2)$, Elena Álvarez Martín (3), Ricard Gènova Maleras (4), y Consuelo \\ Morant Ginestar (5)
}

(1) Investén-ISCIII. Instituto de Salud Carlos III. Ministerio de Ciencia e Innovación. Madrid.

(2) Oficina de Planificación Sanitaria y Calidad. Agencia de Calidad del SNS. Ministerio de Sanidad y Consumo. Madrid.

(3) Departamento de Medicina Preventiva y Salud Pública. Universidad Rey Juan Carlos I. Alcorcón. Madrid.

(4) Servicio de Informes de Salud y Estudios. Consejería de Sanidad. Comunidad de Madrid. Madrid.

(5) Oficina Regional de Coordinación de Salud Mental. Servicio Madrileño de Salud. Consejería de Sanidad. Comunidad de Madrid. Madrid.

\section{RESUMEN}

Fundamento: El Instituto de Salud Carlos III destina parte de sus presupuestos a la financiación de la investigación sanitaria en el ámbito del Sistema Nacional de Salud (SNS). El objetivo del estudio es analizar el grado de correlación de la financiación de la investigación sanitaria en el SNS con el patrón de carga de enfermedad en la población española.

Métodos: Estudio transversal. Se calculan los años de vida ajustados por discapacidad (AVAD), los años de vida perdidos (AVP) y la mortalidad por causa. Se realiza un análisis de correlación (Rho de Spearman) para examinar la asociación entre estas medidas y los fondos de investigación 2006/2007.

Resultados: Por categorías de enfermedad $(n=21)$, la correlación entre la financiación y las medidas de carga de enfermedad es: AVAD $(r=0,72 ; p<0,001)$, mortalidad $(r=0,60 ; p=0,004)$ y AVP $(r=0,56 ; p=0,008)$. A nivel de subcategorías $(\mathrm{n}=52)$ : AVAD $(\mathrm{r}=0,55 ; \mathrm{p}<0,001)$, mortalidad $(\mathrm{r}=0,54 ; \mathrm{p}<0,001)$ y AVP $(\mathrm{r}=0,55 ; \mathrm{p}<0,001)$. Los tumores malignos, las enfermedades neuropsiquiátricas, las cardiovasculares y las infecciosas y parasitarias son las causas con mayor partida presupuestaria asignada. Por otro lado, las enfermedades endocrinas y de la sangre, las infecciosas y parasitarias, y las anomalías congénitas reciben la mayor financiación por AVAD perdido.

Conclusiones: Se observa la existencia de una asociación positiva moderada-alta de las medidas de carga de enfermedad con la financiación de la investigación, si bien existen categorías de enfermedad sobre- o infrafinanciadas en relación con la carga que provocan. En planificación sanitaria, la carga de enfermedad aporta información útil a los debates sobre establecimiento de prioridades en investigación.

Palabras clave: Prioridades en salud. Apoyo a la investigación como asunto. Investigación en servicios de salud. Carga de enfermedad. Años de Vida Ajustados por Discapacidad. AVAD. Años Potenciales de Vida Perdidos. APVP. Mortalidad.

\section{Correspondencia:}

Ferrán Catalá López

Instituto de Salud Carlos III.

Ministerio de Ciencia e Innovación

C/ Sinesio Delgado 6, Pabellón 13. 28029 Madrid.

Correo electrónico: fcatala@isciii.es

\section{ABSTRACT}

\section{Relationship between Research Funding in the Spanish National Health System and the Burden of Disease}

Background: The Carlos III Health Institute (Instituto de Salud Carlos III - Spain) allocates funding to health research support in the Spanish National Health System (NHS). This study aimed to analyse the correlation of health research fund allocations in the NHS and the burden of disease in Spanish population.

Methods: Cross-sectional study. Burden of disease measures were calculated: disability-adjusted life-years (DALYs), years of life lost (YLLs) and mortality by cause. A correlation analysis (Spearman's Rho) was applied to test the association between these measures and 2006/2007 health research funding.

Results: Using disease categories $(n=21)$, the correlation between funding and disease-burden measures is: DALY $(r=0.72 ; \mathrm{p}<0.001)$, mortality $(\mathrm{r}=0.60 ; \mathrm{p}=0.004)$ and YLL $(\mathrm{r}=0.56 ; \mathrm{p}=0.008)$. By disease-specific subcategories $(n=52)$ : DALY $(r=0.55 ; p<0.001)$, mortality $(r=0.54 ; p<0.001)$ and YLL $(r=0.55 ; \mathrm{p}<0.001)$. Malignant neoplasms, neuropsychiatric conditions, cardiovascular diseases and infectious and parasitic diseases receive the greater health research funding support. However, the higher funds allocated per DALY lost ratios were for blood and endocrine disorders, infectious and parasitic diseases and congenital anomalies.

Conclusion: Our analysis suggests that NHS research funding is positive moderately high-associated with the burden of disease in Spain, although there exists certain disease's categories that are over or under-funded in relation to their burden generated. In health planning, burden of disease studies contributes with useful information for setting health research priorities.

Key words: Health priorities. Research support as topic. Health services research. Burden of illness. Disability-adjusted life years. DALY. Potential Years of Life Lost. PYLL. Mortality.

Ninguno de los autores declara tener conflicto de intereses. Ferrán Catalá López está vinculado laboralmente al Instituto de Salud Carlos III a través de la Encomienda de Gestión entre el Ministerio de Sanidad y Consumo (Agencia de Calidad del Sistema Nacional de Salud) y el Instituto de Salud Carlos III para el desarrollo de actividades de formación, investigación y asesoramiento (expediente: DGVI-1273/07-TS-12), área de actividad "Desarrollo del Plan de Calidad del Sistema Nacional de Salud". Estos organismos no comparten necesariamente las opiniones expresadas por los autores, quienes han llevado a cabo los análisis en todo momento con independencia. 


\section{INTRODUCCIÓN}

La investigación sanitaria es un instrumento clave para mejorar la calidad y la expectativa de vida de la población y por ello ha de ser fomentada en todo el sistema sanitario como uno de los elementos fundamentales para su progreso. En este sentido, el Instituto de Salud Carlos III (ISCIII), organismo público de investigación, tiene la responsabilidad de promocionar y fomentar la investigación sanitaria en el Sistema Nacional de Salud $(\mathrm{SNS})^{1}$ y participa en su planificación y priorización. Desde hace algo más de 20 años el ISCIII destina buena parte de sus presupuestos a la financiación de la investigación sanitaria, mediante diferentes fórmulas que quedan actualmente recogidas en los Planes Nacionales de Investigación, Desarrollo e Innovación Tecnológica $(\mathrm{I}+\mathrm{D}+\mathrm{I})^{2,3}$, instrumento de la política científica y tecnológica de la Administración General del Estado. Este fomento de la investigación sanitaria se viene realizando a través de ayudas o subvenciones con cargo a fondos públicos que anualmente convoca el Fondo de Investigación Sanitaria (FIS) del ISCIII. La concesión de estas ayudas se realiza en régimen de concurrencia competitiva y pueden optar a ellas las entidades públicas o privadas sin ánimo de lucro.

En investigación es necesario establecer prioridades, al igual que en otras tantas actividades en las que no se dispone de suficientes recursos para acometer todos los objetivos posibles. El hecho de planificar y establecer prioridades pretende orientar los esfuerzos de la investigación a las necesidades de salud de la población así como ajustarse a los recursos existentes. En la literatura se abordan las dificultades para decidir sobre la asignación de fondos públicos aunque se reconocen distintos procedimientos para guiar la toma de decisiones e informar los debates sobre prioridades $^{4-6}$. En esta línea, los propios planes nacionales han venido justificando desde sus ediciones anteriores el establecimiento de prioridades en torno a problemas de salud que contemplen desde las bases moleculares, genéticas y fisiopatológicas hasta las fases clínicas de diagnóstico y tratamiento, así como aspectos comunitarios y de evaluación de servicios sanitarios, preferentemente de aquellos grupos de entidades cuyo interés social y sanitario sea más relevante, teniendo en cuenta los estudios de carga de enfermedad y de equidad personal ${ }^{2,3}$.

La carga de enfermedad, cuyo indicador son los Años de Vida Ajustados por Discapacidad (AVAD, en inglés Disability-Adjusted Life Years, DALY), mide las pérdidas de salud en la población que representan tanto las consecuencias mortales como las no mortales de las enfermedades, lesiones y los factores de riesgo asociados a las mismas ${ }^{7,8}$. La ventaja de utilizar esta medida sintética de salud de la población en políticas de salud y planificación sanitaria es que ofrece la posibilidad de resumir en un único indicador el conjunto de datos epidemiológicos de cada enfermedad (mortalidad, incidencia, prevalencia, duración, discapacidad, gravedad), pudiendo servir para medir y comparar la salud de diferentes poblaciones o grupos sociales, conocer la evolución de la salud de una población o la magnitud de un problema de salud a lo largo del tiempo, utilizar estos resultados como un instrumento en la definición de prioridades en salud o incluso en la evaluación del impacto de determinadas intervenciones sanitarias a través del empleo de técnicas de análisis de eficiencia (p. ej., análisis coste-efectividad o coste-utilidad) $)^{9-12}$.

En algunos estudios internacionales ${ }^{16-}$ ${ }^{21}$ se ha valorado la actuación de diversos organismos en cuanto a la asignación de recursos públicos destinados a la investigación sanitaria se refiere. Un ejemplo de 
ello fue el trabajo llevado a cabo por Gross y cols. ${ }^{16}$, donde se determinó en qué medida la distribución de los fondos destinados a la investigación de 29 condiciones o enfermedades específicas por parte de los Institutos Nacionales de Salud (en inglés, National Institutes of Health - NIH) de EEUU se asociaba con distintas medidas de carga de enfermedad. Dada la existencia de presupuestos limitados para financiar la investigación de enfermedades con distinta carga sanitaria, económica y social, el interés por este tipo de análisis ha ido aumentando progresivamente en la última década como una forma sistemática de generar conocimiento para orientar futuras agendas de investigación ${ }^{8,13}$.

En este contexto, el objetivo de este trabajo es analizar el grado de correlación de la financiación de la investigación sanitaria en el ámbito del SNS con el patrón de carga de enfermedad de la población española.

\section{MATERIAL Y MÉTODOS}

Se realizó un estudio transversal calculando la carga de enfermedad en la población española y determinando el grado de correlación existente con los fondos asignados a la investigación sanitaria en el ámbito del SNS para el estudio de enfermedades específicas.

\section{Cálculo de la carga de enfermedad en España para el año 2006}

Siguiendo los criterios establecidos por Murray y López en el estudio de Carga Global de Enfermedad ${ }^{8}$ desarrollado por la Organización Mundial de la Salud (OMS), el Banco Mundial y la Universidad de Harvard, el conjunto de enfermedades y lesiones se ha organizado según la Clasificación de Carga de Enfermedad
(CCE) en 3 grandes grupos: $a$ ) enfermedades transmisibles, maternas, perinatales y deficiencias nutricionales; $b$ ) enfermedades no transmisibles, y $c$ ) accidentes $\mathrm{y}$ lesiones. Cada grupo de enfermedades se divide en 21 categorías (tabla 1) que, a su vez, se pueden desagregar en distintas subcategorías. Las causas mal definidas y las rúbricas inespecíficas se han reasignado siguiendo los algoritmos propuestos por Murray y Lopez ${ }^{8}$.

Los AVAD atribuibles a cada enfermedad o lesión son el resultado de sumar el tiempo de vida perdido por muerte prematura -Años de Vida Perdidos (AVP)- y las pérdidas funcionales y de bienestar causadas por la discapacidad y la mala salud -Años Vividos con Discapacidad (AVD)(tabla 2).

Los AVP se calculan multiplicando las defunciones en cada grupo de edad por la esperanza de vida a la edad del fallecimiento. Se utilizaron datos de mortalidad por sexo, edad y causa para el año 2006 procedentes del fichero de microdatos anonimizado del registro de mortalidad del Instituto Nacional de Estadística $(\mathrm{INE})^{14}$. Las defunciones fueron reagrupadas y reasignadas según los criterios de la CCE. El límite de esperanza de vida utilizado viene definido por una tabla de vida estándar ampliamente utilizada en los estudios de carga de enfermedad (Princeton West nivel 26 modificada) ${ }^{15}$. Los AVD, por su parte, se calculan multiplicando la incidencia por la duración media de la enfermedad y por un valor que pondera la gravedad de la discapacidad -escala de 0 (plena salud) a 1 (muerte)-. En este trabajo, como en otros de características similares ${ }^{16-25}$, se ha aplicado un método indirecto de estimación de AVD desarrollado en el estudio de Carga Global de Enfermedad ${ }^{8}$, que requiere la información correspondiente a una población de referencia detallada por sexo, edad y causa para los AVP y AVD, y la 
Tabla 1

Clasificación de carga de enfermedad ( $n=21$ categorías)

\begin{tabular}{|c|c|}
\hline Categorías de enfermedad & Cód. CIE-10 \\
\hline 10100. Infecciosas y parasitarias & A00-B99, G00, G03-G04, N70-N73 \\
\hline 10200. Infecciones respiratorias & J00-J06, J10-J18, J20-J22, H65-H66 \\
\hline 10300. Condiciones maternas & O00-O99 \\
\hline 10400. Condiciones perinatales & P00-P96 \\
\hline 10500. Condiciones nutricionales & E00-E02, E40-E46, E50, D50-D53, D64.9, E51-E64 \\
\hline 20100. Tumores malignos & $\mathrm{C} 00-\mathrm{C} 97$ \\
\hline 20200. Otros tumores & D00-D48 \\
\hline 20300. Diabetes mellitus & E10-E14 \\
\hline 20400. Endocrinas y de la sangre & D55-D64 (excepto D64.9), D65-D89, E03-E07, E15-E16, E20-E34, E65-E88 \\
\hline 20500. Neuropsiquiátricas & F01-F99, G06-G98 \\
\hline 20600. Órganos de los sentidos & H00-H61, H68-H93 \\
\hline 20700. Cardiovasculares & I00-199 \\
\hline 20800. Respiratorias & J30-J98 \\
\hline 20900. Aparato digestivo & K20-K92 \\
\hline 21000. Aparato genito-urinario & N00-N64, N75-N98 \\
\hline 21100. Piel & L00-L98 \\
\hline 21200. Osteomusculares y tejido conectivo & M00-M99 \\
\hline 21300. Anomalías congénitas & Q00-Q99 \\
\hline 21400. Condiciones orales & K00-K14 \\
\hline 30100. Accidentes y lesiones no intencionales & V01-X59, Y40-Y86, Y88, Y89 \\
\hline 30200. Accidentes y lesiones intencionales & X60-Y09, Y35-Y36, Y870, Y871 \\
\hline
\end{tabular}

Adaptada a partir de Mathers CD y cols. (2004).

estructura por sexo y edad de dicha población, además de los AVP de la población cuya carga de enfermedad se pretende calcular. Se ha tomado para ello la subregión europea de muy baja mortalidad (Euro-A) definida por la $\mathrm{OMS}^{26}$, a la que pertenece España. Se ha utilizado

Tabla 2

Fórmulas simplificadas para el cálculo de los Años de Vida Ajustados por Discapacidad-AVAD-

$A V A D=A V P+A V D$
$A V P=\sum_{0}^{L} d_{i} * e_{i}$
$A V D=\sum_{0}^{L} N_{i} * I_{i} * T_{i} * D$

AVP, Años de Vida Perdidos; d, número de defunciones en cada grupo de edad; e, esperanza de vida a la edad de muerte en cada grupo de edad. AVD, Años Vividos con Discapacidad; N, población susceptible de contraer la enfermedad en cada grupo de edad; I, tasa de incidencia anual de la enfermedad en cada grupo de edad; T, tiempo de duración de la enfermedad (en años) en cada grupo de edad; D, grado de discapacidad asignado a cada enfermedad (estado de salud ideal $=0$; muerte $=1$ ); L, esperanza de vida estándar obtenida de la tabla modelo de baja mortalidad Princeton West nivel 26. la información correspondiente a la última estimación realizada por la OMS en el momento del análisis ${ }^{28}$, aplicando a los AVP calculados directamente para la población española la razón de AVD/AVP por causa, sexo y edad correspondiente a la subregión Euro-A. Para aquellas causas que tienen muy baja mortalidad pero alta discapacidad en las que la razón AVD/AVP es muy volátil, el método seguido sugiere estimar los AVD aplicando la tasa de AVD de la población de referencia (Euro-A) a la población objeto de estudio (España). La población española utilizada fue la estimación del INE a 1 de julio de 2006, con un total de 44.068.244 personas $^{27}$. Al igual que en el estudio mundial de Carga Global de Enferme$\operatorname{dad}^{8,28}$ se han incorporado a los cálculos las siguientes valoraciones sociales: una tasa de descuento del 3\% (preferencia temporal), lo que hace que un beneficio presente sea más valioso que el mismo 
beneficio obtenido en el futuro, y una ponderación del valor de los años en función de la edad $(\mathrm{K}=1)$, atribuyendo mayor peso a las muertes o discapacidades en los adultos jóvenes. Se utilizó el programa informático Gesmor ${ }^{29}$.

\section{Análisis de proyectos y distribución de los fondos asignados al estudio de enfermedades}

Fueron objeto de estudio las ayudas concedidas para la realización de proyectos y estudios a través del Programa de Promoción de la Investigación Biomédica y en Ciencias de la Salud durante las convocatorias correspondientes a los años 2006 y 2007 en el marco del Plan Nacional de I+D+I 2004-2007 30, 31. Concretamente se incluyeron: a) proyectos de investigación $\left.{ }^{32}, 33 ; b\right)$ estudios de investigaciones sobre evaluación de tecnologías sanitarias e investigación en servicios de salud ${ }^{34,35}, \mathrm{y} c$ ) proyectos de investigación clínica de carácter no comercial con medicamentos de uso humano ${ }^{36}$.

Se seleccionaron los proyectos evaluados y aprobados en las dos últimas convocatorias a través de su identificación a partir de los registros de los que se dispone (bases de datos del FIS). Se procedió a una revisión sistemática con el fin de clasificar los proyectos (y los fondos asignados) dentro de cada uno de los grupos, categorías y subcategorías de la CCE. Si bien la etapa de revisión y clasificación fue realizada por epidemiólogos, en ocasiones fue requerida la opinión de expertos clínicos. Los proyectos fueron clasificados de acuerdo al título del estudio, atendiendo a la causa de enfermedad principal. Para aquellos proyectos que pudieran ser clasificados en más de una causa de enfermedad, la financiación se distribuyó proporcionalmente entre las patologías implicadas. Este criterio era válido entre las distintas categorías y subcategorías, y ha sido utilizado en otros estudios publicados ${ }^{16,19}$.
Finalmente, se obtuvo la razón fondos de investigación asignados (en euros) por cada AVAD perdido, como resultado de dividir el total de la financiación destinada a la investigación de las distintas categorías/subcategorías de enfermedades entre el número total de AVAD perdidos correspondientes a cada una de ellas.

\section{Análisis estadístico}

Se realizó un análisis de correlación bivariado a través del paquete estadístico SPSS 15.0, utilizando el coeficiente de correlación no paramétrico de Spearman (Rho) para examinar la dirección y fuerza de asociación lineal entre variables cuantitativas en escala proporcional (las medidas de carga de enfermedad y la financiación de la investigación sanitaria). El nivel de significación estadística se estableció en un valor de 0,05 (nivel de confianza del 95\%).

\section{RESULTADOS}

\section{Carga de enfermedad en España en el año 2006}

En España, se estima que en 2006 se perdieron 5.025.472 AVAD, con una tasa de 11.404 AVAD por cada 100 mil habitantes. De ellos, el 57,7\% (2.901.871 AVD) se ocasionaron por discapacidad o mala salud y el $42,3 \%$ (2.123.602 AVP) por mortalidad prematura. Las principales causas en número de AVAD fueron las enfermedades neuropsiquiátricas (con un 31,8\% sobre el total), por delante de los tumores malignos $(15,9 \%)$, las enfermedades cardiovasculares $(12,3 \%)$ o las enfermedades respiratorias $(7,5 \%)$. Entre las subcategorías de causas de enfermedad, destaca el peso de los AVAD en la depresión unipolar $(8,8 \%)$, las demencias $(7,6 \%)$, la cardiopatía isquémica (4,5\%), el abuso de alcohol $(4,1 \%)$, el cáncer de pulmón $(3,4 \%)$ o la enfermedad cerebrovascular $(2,9 \%)$, como principales causas de carga de enfermedad (tabla 3 ). 
Tabla 3

Indicadores de carga de enfermedad y financiación de la investigación, por categorías $(\mathrm{n}=21)$ y subcategorías seleccionadas* $(\mathrm{n}=\mathbf{5 2})$

\begin{tabular}{|c|c|c|c|c|c|c|c|c|c|c|}
\hline Cód. & $\begin{array}{c}\text { Clasificación } \\
\text { de enfermedades }\end{array}$ & $\begin{array}{l}\text { AVAD } \\
\text { (miles) }\end{array}$ & $\%$ & $\begin{array}{c}\text { AVP } \\
\text { (miles) }\end{array}$ & $\%$ & $\begin{array}{l}\text { Mortalidad } \\
\text { (miles) }\end{array}$ & $\%$ & $\begin{array}{c}\text { Fondos } \\
(\text { miles de } € \text { ) }\end{array}$ & $\%$ & $\begin{array}{c}\text { Fondos }(€) \\
\text { por AVAD }\end{array}$ \\
\hline & $\begin{array}{l}\text { Transmisibles, maternas, } \\
\text { perinatales y nutricionales }\end{array}$ & 247,7 & 4,9 & 133,1 & 6,3 & 17,6 & 4,7 & $17.474,9$ & 13,9 & 70,6 \\
\hline \multirow[t]{7}{*}{10100} & Infecciosas y parasitarias & 113,7 & 2,3 & 66,9 & 3,1 & 7,4 & 2,0 & $12.344,9$ & 9,8 & 108,6 \\
\hline & VIH/SIDA & 36,9 & 0,7 & 28,1 & 1,3 & 1,4 & 0,4 & $4.026,5$ & 3,2 & 109,1 \\
\hline & Diarreas & 11,5 & 0,2 & 1,5 & 0,1 & 0,4 & 0,1 & 975,4 & 0,8 & 84,8 \\
\hline & Hepatitis B y C & 10,3 & 0,2 & 8,9 & 0,4 & 1,0 & 0,3 & 974,4 & 0,8 & 95,0 \\
\hline & ETS & 8,9 & 0,2 & 0,2 & 0,0 & 0,0 & 0,0 & 22,6 & 0,0 & 2,5 \\
\hline & Meningitis & 4,9 & 0,1 & 3,0 & 0,1 & 0,2 & 0,1 & 277,1 & 0,2 & 56,2 \\
\hline & Tuberculosis & 4,6 & 0,1 & 2,9 & 0,1 & 0,4 & 0,1 & 840,4 & 0,7 & 182,0 \\
\hline \multirow[t]{4}{*}{10200} & Infecciones respiratorias & 36,6 & 0,7 & 31,6 & 1,5 & 8,8 & 2,4 & $2.215,5$ & 1,8 & 60,6 \\
\hline & Vías respiratorias bajas & 32,7 & 0,7 & 31,3 & 1,5 & 8,8 & 2,4 & $1.693,4$ & 1,3 & 51,7 \\
\hline & Otitis media & 3,2 & 0,1 & 0,0 & 0,0 & 0,0 & 0,0 & 36,3 & 0,0 & 11,3 \\
\hline & Vías respiratorias altas & 0,6 & 0,0 & 0,3 & 0,0 & 0,1 & 0,0 & 6,1 & 0,0 & 10,0 \\
\hline 10300 & Condiciones maternas & 18,2 & 0,4 & 0,4 & 0,0 & 0,0 & 0,0 & $1.569,5$ & 1,2 & 86,3 \\
\hline 10400 & Condiciones perinatales & 49,2 & 1,0 & 33,2 & 1,6 & 1,0 & 0,3 & 958,5 & 0,8 & 19,5 \\
\hline \multirow[t]{2}{*}{10500} & Deficiencias nutricionales & 30,1 & 0,6 & 1,1 & 0,1 & 0,3 & 0,1 & 386,5 & 0,3 & 12,8 \\
\hline & No transmisibles & $4.441,0$ & 88,4 & $1.748,8$ & 82,4 & 337,8 & 90,9 & $107.085,1$ & 85,2 & 24,1 \\
\hline \multirow[t]{22}{*}{20100} & Tumores malignos & 797,7 & 15,9 & 720,2 & 33,9 & 101,0 & 27,2 & $26.992,3$ & 21,5 & 33,8 \\
\hline & Pulmón & 171,4 & 3,4 & 166,4 & 7,8 & 21,1 & 5,7 & $1.008,7$ & 0,8 & 5,9 \\
\hline & Colon/recto & 101,3 & 2,0 & 84,0 & 4,0 & 14,3 & 3,8 & $1.865,1$ & 1,5 & 18,4 \\
\hline & Mama & 70,6 & 1,4 & 58,2 & 2,7 & 6,6 & 1,8 & $3.856,6$ & 3,1 & 54,6 \\
\hline & Estómago & 42,4 & 0,8 & 40,6 & 1,9 & 6,2 & 1,7 & 466,5 & 0,4 & 11,0 \\
\hline & Páncreas & 37,9 & 0,8 & 36,9 & 1,7 & 5,3 & 1,4 & 464,6 & 0,4 & 12,3 \\
\hline & Linfoma, mieloma & 33,7 & 0,7 & 31,9 & 1,5 & 4,5 & 1,2 & $1.508,3$ & 1,2 & 44,8 \\
\hline & Hígado & 32,5 & 0,6 & 31,9 & 1,5 & 4,8 & 1,3 & 646,3 & 0,5 & 19,9 \\
\hline & Vejiga & 31,9 & 0,6 & 25,2 & 1,2 & 4,9 & 1,3 & 942,7 & 0,8 & 29,5 \\
\hline & Encéfalo & 30,7 & 0,6 & 29,9 & 1,4 & 2,7 & 0,7 & $1.133,7$ & 0,9 & 37,0 \\
\hline & Leucemia & 28,9 & 0,6 & 27,6 & 1,3 & 3,4 & 0,9 & $2.149,6$ & 1,7 & 74,5 \\
\hline & Próstata & 27,5 & 0,5 & 21,8 & 1,0 & 5,8 & 1,6 & 813,5 & 0,6 & 29,6 \\
\hline & Boca, orofaringe & 23,9 & 0,5 & 22,3 & 1,1 & 2,3 & 0,6 & 80,3 & 0,1 & 3,4 \\
\hline & Ovario & 19,1 & 0,4 & 16,8 & 0,8 & 1,9 & 0,5 & 444,6 & 0,4 & 23,3 \\
\hline & Cuerpo uterino & 19,1 & 0,4 & 9,5 & 0,4 & 1,5 & 0,4 & 451,4 & 0,4 & 23,6 \\
\hline & Esófago & 16,5 & 0,3 & 16,0 & 0,8 & 1,9 & 0,5 & 72,0 & 0,1 & 4,4 \\
\hline & Riñón & 15,9 & 0,3 & 14,7 & 0,7 & 2,1 & 0,6 & 233,3 & 0,2 & 14,7 \\
\hline & Laringe & 14,6 & 0,3 & 13,7 & 0,6 & 1,7 & 0,4 & 93,8 & 0,1 & 6,4 \\
\hline & Melanoma & 11,9 & 0,2 & 11,0 & 0,5 & 1,4 & 0,4 & 909,7 & 0,7 & 76,6 \\
\hline & Cuello uterino & 9,5 & 0,2 & 7,7 & 0,4 & 0,7 & 0,2 & 936,0 & 0,7 & 98,7 \\
\hline & Hueso y cartílagos & 5,8 & 0,1 & 5,5 & 0,3 & 0,3 & 0,1 & 200,6 & 0,2 & 34,7 \\
\hline & Tiroides & 2,0 & 0,0 & 1,8 & 0,1 & 0,3 & 0,1 & 73,2 & 0,1 & 37,1 \\
\hline 20200 & Otros tumores & 21,4 & 0,4 & 21,4 & 1,0 & 3,5 & 0,9 & 182,1 & 0,1 & 8,5 \\
\hline 20300 & Diabetes mellitus & 84,4 & 1,7 & 39,3 & 1,8 & 10,0 & 2,7 & $2.726,7$ & 2,2 & 32,3 \\
\hline 20400 & Endocrinas y de la sangre & 59,5 & 1,2 & 19,4 & 0,9 & 2,8 & 0,8 & $6.863,9$ & 5,5 & 115,3 \\
\hline \multirow[t]{5}{*}{20500} & Neuropsiquiátricas & $1.599,5$ & 31,8 & 110,9 & 5,2 & 28,7 & 7,7 & $21.248,7$ & 16,9 & 13,3 \\
\hline & Depresión unipolar & 444,7 & 8,8 & 0,2 & 0,0 & 0,1 & 0,0 & $1.779,5$ & 1,4 & 4,0 \\
\hline & Demencias & 381,3 & 7,6 & 56,0 & 2,6 & 21,9 & 5,9 & $2.473,8$ & 2,0 & 6,5 \\
\hline & Abuso de alcohol & 208,4 & 4,1 & 3,6 & 0,2 & 0,3 & 0,1 & 31,8 & 0,0 & 0,2 \\
\hline & Migraña & 79,6 & 1,6 & 0,0 & 0,0 & 0,0 & 0,0 & 222,0 & 0,2 & 2,8 \\
\hline
\end{tabular}


Tabla 3 (continuación)

Indicadores de carga de enfermedad y financiación de la investigación, por categorías $(n=21)$ y subcategorías seleccionadas* $(n=52)$

\begin{tabular}{|c|c|c|c|c|c|c|c|c|c|c|}
\hline Cód. & $\begin{array}{c}\text { Clasificación } \\
\text { de enfermedades }\end{array}$ & $\begin{array}{l}\text { AVAD } \\
\text { (miles) }\end{array}$ & $\%$ & $\begin{array}{c}\text { AVP } \\
\text { (miles) }\end{array}$ & $\%$ & $\begin{array}{l}\text { Mortalidad } \\
\text { (miles) }\end{array}$ & $\%$ & $\begin{array}{c}\text { Fondos } \\
(\text { miles de } € \text { ) }\end{array}$ & $\%$ & $\begin{array}{c}\text { Fondos }(€) \\
\text { por AVAD }\end{array}$ \\
\hline \multirow[t]{7}{*}{20500} & Adicción a drogas & 70,0 & 1,4 & 2,3 & 0,1 & 0,1 & 0,0 & $1.423,6$ & 1,1 & 20,3 \\
\hline & Trastornos bipolares & 68,3 & 1,4 & 0,1 & 0,0 & 0,0 & 0,0 & 198,9 & 0,2 & 2,9 \\
\hline & Esquizofrenia & 64,1 & 1,3 & 1,0 & 0,0 & 0,1 & 0,0 & $2.291,3$ & 1,8 & 35,8 \\
\hline & Enfermedad de Parkinson & 30,2 & 0,6 & 8,5 & 0,4 & 2,6 & 0,7 & 979,4 & 0,8 & 32,4 \\
\hline & $\begin{array}{l}\text { Trastorno } \\
\text { obsesivo-compulsivo }\end{array}$ & 28,7 & 0,6 & 0,0 & 0,0 & 0,0 & 0,0 & 362,5 & 0,3 & 12,6 \\
\hline & Epilepsia & 13,9 & 0,3 & 4,8 & 0,2 & 0,4 & 0,1 & 290,2 & 0,2 & 20,9 \\
\hline & Esclerosis múltiple & 10,1 & 0,2 & 2,3 & 0,1 & 0,2 & 0,0 & 945,8 & 0,8 & 93,2 \\
\hline \multirow[t]{2}{*}{20600} & Órganos de los sentidos & 260,0 & 5,2 & 0,0 & 0,0 & 0,0 & 0,0 & $3.704,8$ & 2,9 & 14,3 \\
\hline & Glaucoma & 11,1 & 0,2 & 0,0 & 0,0 & 0,0 & 0,0 & 269,8 & 0,2 & 24,3 \\
\hline \multirow[t]{5}{*}{20700} & Cardiovasculares & 618,2 & 12,3 & 517,9 & 24,4 & 124,8 & 33,6 & $19.865,8$ & 15,8 & 32,1 \\
\hline & Cardiopatía isquémica & 227,0 & 4,5 & 194,5 & 9,2 & 38,5 & 10,4 & $3.651,3$ & 2,9 & 16,1 \\
\hline & Cerebrovascular & 145,1 & 2,9 & 130,3 & 6,1 & 34,0 & 9,2 & $2.947,1$ & 2,3 & 20,3 \\
\hline & Inflamatoria del corazón & 50,2 & 1,0 & 30,4 & 1,4 & 6,3 & 1,7 & 777,8 & 0,6 & 15,5 \\
\hline & Hipertensiva & 26,1 & 0,5 & 21,2 & 1,0 & 6,9 & 1,9 & 658,9 & 0,5 & 25,2 \\
\hline \multirow[t]{3}{*}{20800} & Respiratorias & 377,4 & 7,5 & 123,7 & 5,8 & 31,7 & 8,5 & $5.822,2$ & 4,6 & 15,4 \\
\hline & EPOC & 114,4 & 2,3 & 56,8 & 2,7 & 16,6 & 4,5 & $2.418,4$ & 1,9 & 21,1 \\
\hline & Asma & 67,4 & 1,3 & 4,0 & 0,2 & 0,8 & 0,2 & $1.036,8$ & 0,8 & 15,4 \\
\hline \multirow[t]{2}{*}{20900} & Aparato digestivo & 225,9 & 4,5 & 120,8 & 5,7 & 20,0 & 5,4 & $6.475,0$ & 5,2 & 28,7 \\
\hline & Cirrosis & 61,4 & 1,2 & 49,6 & 2,3 & 4,9 & 1,3 & $2.336,9$ & 1,9 & 38,0 \\
\hline \multirow[t]{2}{*}{21000} & Genito-urinarias & 63,8 & 1,3 & 33,0 & 1,6 & 9,7 & 2,6 & $3.819,8$ & 3,0 & 59,9 \\
\hline & Nefritis, nefrosis & 24,3 & 0,5 & 23,1 & 1,1 & 6,6 & 1,8 & $2.270,3$ & 1,8 & 93,4 \\
\hline 21100 & Piel & 10,4 & 0,2 & 3,4 & 0,2 & 1,1 & 0,3 & 182,0 & 0,1 & 17,5 \\
\hline \multirow[t]{3}{*}{21200} & $\begin{array}{l}\text { Osteomusculares y tejido } \\
\text { conectivo }\end{array}$ & 234,8 & 4,7 & 11,4 & 0,5 & 3,5 & 0,9 & $4.394,1$ & 3,5 & 18,7 \\
\hline & Artrosis & 124,0 & 2,5 & 0,2 & 0,0 & 0,1 & 0,0 & 549,6 & 0,4 & 4,4 \\
\hline & Artritis reumatoide & 41,5 & 0,8 & 1,2 & 0,1 & 0,2 & 0,1 & $1.458,7$ & 1,2 & 35,1 \\
\hline 21300 & Anomalías congénitas & 49,3 & 1,0 & 27,2 & 1,3 & 1,0 & 0,3 & $4.726,5$ & 3,8 & 95,8 \\
\hline \multirow[t]{2}{*}{21400} & Condiciones orales & 38,8 & 0,8 & 0,1 & 0,0 & 0,0 & 0,0 & 81,3 & 0,1 & 2,1 \\
\hline & Accidentes y lesiones & 336,8 & 6,7 & 241,6 & 11,4 & 16,1 & 4,3 & $1.066,1$ & 0,8 & 3,2 \\
\hline 30100 & No intencionales & 268,9 & 5,4 & 180,7 & 8,5 & 12,5 & 3,4 & 760,8 & 0,6 & 2,8 \\
\hline \multirow[t]{2}{*}{30200} & Intencionales & 67,8 & 1,3 & 60,9 & 2,9 & 3,6 & 1,0 & 305,4 & 0,2 & 4,5 \\
\hline & Totales & $5.025,5$ & 100 & $2.123,6$ & 100 & 371,5 & 100 & $125.626,2$ & 100 & 25,0 \\
\hline
\end{tabular}

* Las subcategorías seleccionadas no comprenden el total de enfermedades de cada categoría, por lo que no suman el $100 \%$ de la categoría a la que pertenecen.

AVAD, Años de vida ajustados por discapacidad; AVP, Años de vida perdidos; Mortalidad, expresada como el número de defunciones por causa; VIH/SIDA, Virus de Inmunodeficiencia Humana - Síndrome de Inmunodeficiencia Adquirida; ETS, Enfermedades de Transmisión Sexual; EPOC, Enfermedad Pulmonar Obstructiva Crónica.

\section{Financiación de la investigación sanitaria 2006/07 destinada al estudio de enfermedades}

Se revisó un total de 1.615 proyectos, de los cuales el $87,3 \%(\mathrm{n}=1.410)$ fueron finalmente incluidos en el análisis. De éstos, el 3,8\% (n=53) compartieron más de una categoría/subcategoría de enfermedad.
Se excluyeron 205 proyectos por no estar dirigidos al estudio de enfermedades específicas.

Según los datos aportados por el FIS, en el período de estudio fueron asignados 139,6 millones de euros (82,3 millones en 2007 y 57,3 millones en 2006) en concepto de ayudas destinadas a la realización de 
Figura 1

Gráfico de dispersión representando los Años de Vida Ajustados por Discapacidad y los fondos de investigación para 21 categorías de enfermedad

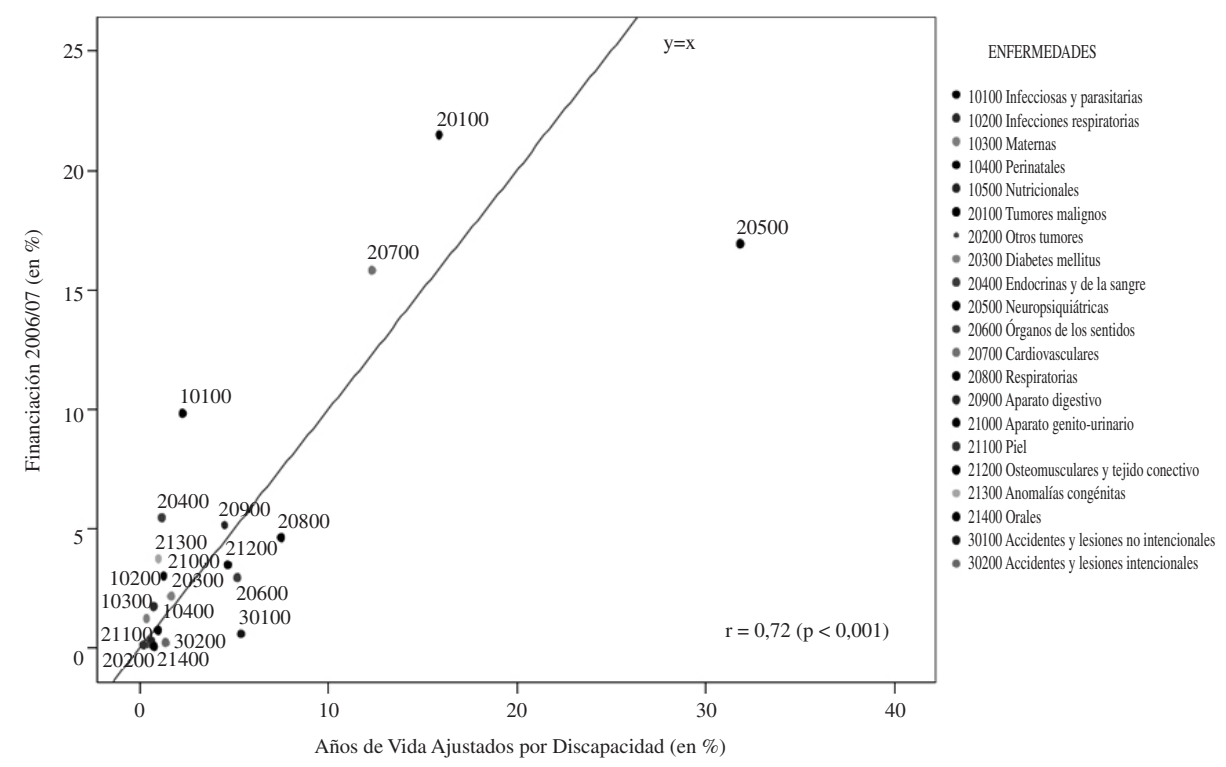

Figura 2

Gráfico de dispersión representando los Años de Vida Perdidos y los fondos de investigación para 21 categorías de enfermedad

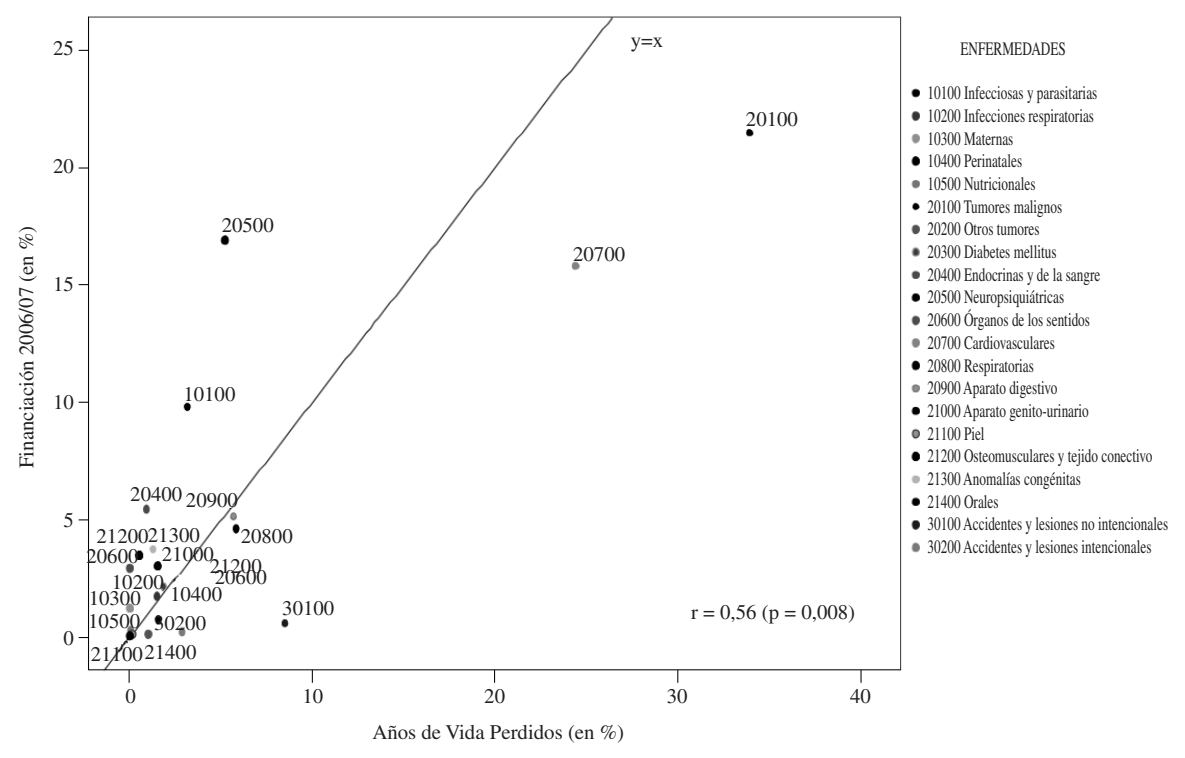


proyectos y/o estudios, de los cuales el 90,0\% (125,6 millones de euros) pudo incluirse y clasificarse dentro de cada una de las categorías $(n=21)$ y subcategorías $(n=52)$ específicas de enfermedad (tabla 3). Por categorías de enfermedad fueron los tumores malignos, las enfermedades neuropsiquiátricas, las enfermedades cardiovasculares y las enfermedades infecciosas y parasitarias las causas con mayor partida presupuestaria asignada $(64,0 \%$ sobre el total). Las subcategorías que mayor financiación recibieron fueron el VIH/SIDA $(3,2 \%)$, el cáncer de mama $(3,1 \%)$, la cardiopatía isquémica $(2,9 \%)$ y la enfermedad cerebrovascular $(2,3 \%)$. Entre las causas seleccionadas para este estudio, las enfermedades de las vías respiratorias altas, las enfermedades de transmisión sexual y el abuso de alcohol fueron las rúbricas específicas con menor inversión en el período analizado.

\section{Asociación entre la carga de enfermedad y los fondos de investigación sanitaria}

Por categorías de enfermedad, se observó una alta correlación entre la financiación y los indicadores de carga de enfermedad con una asociación de signo positivo dentro del nivel de significación estadística establecido (figuras 1-2). Así, la correlación entre los fondos de investigación y los AVAD fue de 0,72 ( $\mathrm{p}<0,001)$, siendo ésta la correlación más alta encontrada. De igual manera, se encontró aso-

Figura 3

Gráficos de dispersión representando medidas de carga de enfermedad y los fondos de investigación para 52 subcategorías de enfermedad
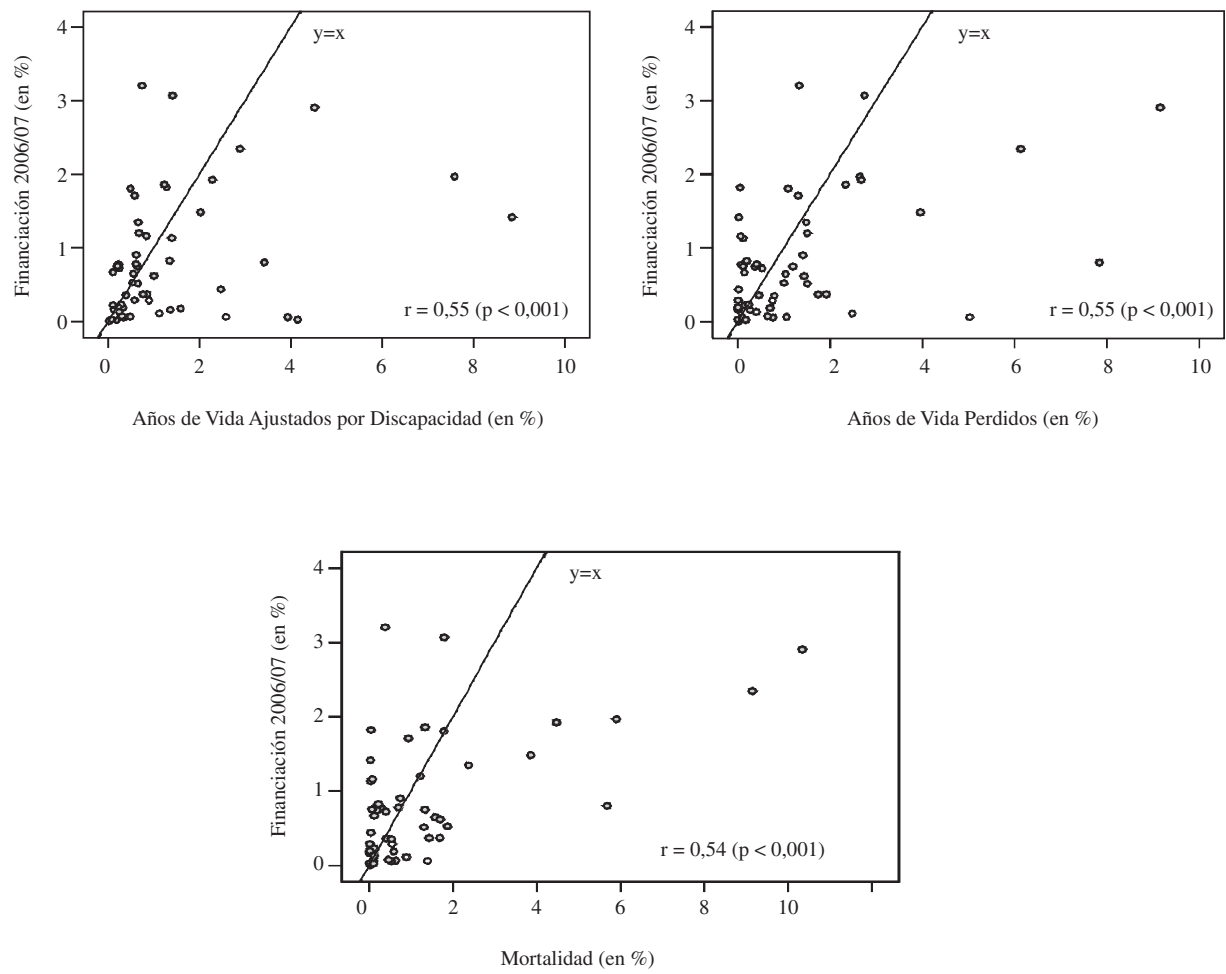
ciación para otros indicadores como los AVP y la mortalidad proporcional, con unos coeficientes de correlación de 0,56 $(\mathrm{p}=0,008)$ y $0,60 \quad(\mathrm{p}=0,004)$, respectivamente. En el análisis de las principales subcategorías de enfermedad también se mostró una asociación positiva (figura 3), moderada y estadísticamente significativa: $0,54(p<0,001)$ en el caso de la mortalidad proporcional, y $0,55(\mathrm{p}<0,001)$ para los AVAD y los AVP.

Las enfermedades endocrinas y de la sangre, las enfermedades infecciosas y parasitarias, y las anomalías congénitas fueron las categorías de enfermedad que mayor financiación recibieron por cada AVAD perdido (ver tabla 3 ). Por subcategorías, cabe destacar que la tuberculosis, el VIH/SIDA, el cáncer de cuello uterino, la hepatitis B y C, las nefropatías o la esclerosis múltiple fueron aquellas causas específicas con mayor inversión por cada AVAD perdido.

Por el contrario, algunas enfermedades neuropsiquiátricas (p.ej., abuso de alcohol, migrañas, trastornos bipolares, depresión), algunos cánceres específicos (p.ej., orales, faringoesofágicos, pulmón), los accidentes y lesiones no intencionales o las enfermedades de transmisión sexual, fueron las causas que tuvieron razones de fondos de investigación asignados por cada AVAD perdido menores.

\section{DISCUSIÓN}

Según el actual Plan Nacional de I+D+I 2008-2011 3 y el Plan de Calidad del SNS ${ }^{37}$, en España, la carga de enfermedad debería ser un criterio utilizado para establecer prioridades en el SNS. Este es el primer trabajo realizado en el ámbito del SNS que evalúa resultados del establecimiento de prioridades en investigación sanitaria a través de métodos objetivos de valoración de carga de enfermedad, lo cual ha permitido observar la existencia de una asociación positiva moderada-alta de estas medidas de salud con la financiación de la investigación del ISCIII. Estos resultados estarían en consonancia con estudios anteriores realizados en países como EE.UU. ${ }^{16,17}$ (National Institutes of Health - NIH, Centers for Disease Control and Prevention - CDC), Canadá ${ }^{18}$ (Medical Research Council of Canada - MRCC), Australia ${ }^{19}$ (National Health and Medical Research Council - NHMRC) o Reino Unido ${ }^{20,21}$ (National Cancer Research Institute - NCRI, UK Clinical Research Collaboration- UKCRC), aunque los diferentes períodos analizados y algunas diferencias metodológicas dificultan la comparación directa entre trabajos.

La planificación en investigación es un proceso complejo, dinámico y evaluable, en el que concurren factores clínico-epidemiológicos, socioeconómicos, políticos, etc. Para establecer prioridades puede recurrirse principalmente a dos tipos de enfoques $^{38}$ : las evaluaciones técnicas a través de datos epidemiológicos o socioeconómicos utilizando métodos cuantitativos (p.ej., estudios de carga de enfermedad), y las aproximaciones subjetivas, a partir de valoraciones implícitas de revisores empleando métodos cualitativos de consenso u otras técnicas grupales, de consulta a agentes clave, etc. ${ }^{39}$ Cada uno de los métodos propuestos en la literatura tiene sus ventajas e inconvenientes, pero "la transparencia y la corresponsabilidad en el proceso resultan favorecidas si los criterios son explícitos y se involucra a los distintos agentes implicados: políticos, gestores, profesionales, investigadores, pacientes y ciudadanos" 40 . Generalmente, las acciones relacionadas con estos procesos requieren del conocimiento del estado de salud de la población. La información de los estudios de carga de enfermedad posibilita que se destinen fondos a unos objetivos predefinidos utilizando una metodología concreta $y$, en nuestra opinión, fundamentada desde la perspectiva de la salud pública. 
Si bien la información de estos estudios puede utilizarse como unidad de resultado en salud de análisis explícitos del costeefectividad (o coste-utilidad), la carga de enfermedad por sí sola no permite la medición de la eficiencia en la asignación de los recursos destinados a la investigación a corto-medio plazo. Por tanto, que la carga de enfermedad sea mayor para una condición no implica necesariamente unos mayores beneficios en salud asignando más recursos, del mismo modo que la existencia de intervenciones sanitarias eficaces no garantiza una mejor utilización de las mismas en términos de efectividad, eficiencia o equidad y accesibilidad a las prestaciones sanitarias. Así, sería conveniente considerar algunas de estas variables así como las ganancias potenciales atribuidas a la investigación pudiendo para ello emplearse el número de AVAD evitados u otros índices alternativos $^{41,42}$. A pesar de las limitaciones y dificultades en la medición de estos resultados, la explicación de algunos de estos aspectos podría contribuir a un mejor conocimiento de la investigación realizada en el ámbito del SNS. En términos económicos, la productividad marginal de las inversiones en investigación e innovación podría ser también un aspecto a valorar.

En otros trabajos publicados, donde el rango de variación en los datos era amplio, se planteó la transformación de las variables a escalas logarítmicas y se utilizaron pruebas de ajuste a la normalidad en la distribución de las variables con la intención de aplicar un análisis de regresión que permitiera la obtención de proyecciones a partir de valores observados para establecer futuras necesidades de financiación, asumiendo la adopción de la carga de enfermedad como único criterio en las decisiones sobre financiación. Este último punto transciende los objetivos de este trabajo concreto, que se limitó a correlacionar las distribuciones relativas de ambas variables, financiación y carga de enfermedad, aunque los métodos propuestos por otros auto- res ${ }^{16,17}$ serán aplicados en futuros análisis para identificar necesidades en determinadas categorías de enfermedad o áreas temáticas y proveer de información complementaria en el establecimiento de prioridades sobre la asignación y reorientación de los fondos de investigación.

Algunas de las limitaciones de estos estudios tienen que ver con las propias de los parámetros utilizados para el cálculo de AVAD, como son las preferencias sociales para establecer el peso de la discapacidad, la tasa de descuento a aplicar y la ponderación del valor de los años en función de la edad. El fundamento para incluir estos parámetros ha sido ampliamente debatido en la literatura científica ${ }^{43-47}$. La valoración del nivel de incertidumbre para estas variables se realizó mediante un análisis de sensibilidad en base a las recomendaciones propuestas por la OMS en el estudio de Carga Global de Enfermedad ${ }^{8,28}$. Esto permitió comprobar la robustez del modelo de carga de enfermedad ante cambios en parámetros como la tasa de descuento (p.ej., sin aplicar descuento) y la ponderación del peso por edades (p.ej., sin ponderación; $\mathrm{K}=0$ ).

El análisis de los AVAD estimados para la población española conjuntamente con la financiación permite afirmar que existen grupos de categorías que reciben más fondos que carga provocan. En general, éstas coinciden con las principales causas de mortalidad. Es el caso, por ejemplo, de los tumores malignos y las enfermedades cardiovasculares que representan el 28,2\% de la carga de enfermedad y el $37,3 \%$ de financiación. Algo similar sucede con las enfermedades infecciosas y parasitarias $(2,3 \%$ sobre el total de AVAD) o las enfermedades endocrinas y de la sangre (1,2\% de AVAD), con el $9,8 \%$ y $5,5 \%$ de la financiación recibida, respectivamente. Todo lo contrario sucede con las enfermedades neuropsiquiátricas, primera causa de carga de enfermedad $(31,8 \%$ sobre el total de AVAD) y 
donde la discapacidad o mala salud $(93,1 \%$ AVD) es mucho mayor a la mortalidad (6,9\% AVP), y que aun siendo la segunda categoría receptora en fondos, le correspondió el $16,9 \%$ sobre el total de la financiación.

En lo referente a la financiación de la investigación realizada en el entorno del SNS, excluida la investigación clínica financiada por la industria farmacéutica y de productos sanitarios, procede mayoritariamente del ISCIII que actúa como organismo financiador a través del FIS. En nuestra opinión, las actividades objeto de este estudio pueden considerarse representativas de las acciones de fomento de la investigación sanitaria realizada en el SNS, si bien en el futuro podría ser interesante ampliar el número de convocatorias correspondientes a la concesión de estas ayudas o incorporar aquellas destinadas a la promoción y consolidación de las estructuras estables de investigación cooperativa y centros de investigación biomédica en red, u otras partidas que incorporasen otras acciones distintas de las propias dirigidas al SNS (p.ej., proyectos de investigación fundamental, proyectos de investigación aplicada, proyectos de innovación, etc.) que quedan recogidas en otros programas del Plan Nacional de $\mathrm{I}+\mathrm{D}+\mathrm{I}^{2,3}$, así como la realización de análisis sobre la distribución de fondos por áreas temáticas (investigación básica, clínica o en epidemiología y salud pública).

Existen otro tipo de convocatorias que no han sido aquí consideradas (p.ej., ayudas concedidas por las distintas Comunidades Autónomas, programas europeos u otras procedentes de otros organismos públicos-privados sin ánimo de lucro, etc.). Este último punto remite a una nueva valoración. La comprensión explícita de cómo éstas acciones, conjuntamente con las que se llevan a cabo desde el sector privado, influyen en la capacidad del SNS para lograr sus objetivos, y cómo las relaciones o acuerdos intersectoriales pueden dar lugar a diversas interacciones, debe ser objeto de reflexión planteándose la siguiente cuestión: ¿debería la financiación pública ajustarse a la distribución de AVAD o debería hacerlo el total de la investigación desarrollada por los sectores público y privado? O incluso: ¿en qué medida la financiación pública debe cubrir la investigación e innovación considerada prioritaria socialmente pero a la que el mercado no es capaz de asignar recursos porque percibe que las ganancias potenciales no justifican el coste de oportunidad de la inversión? Este punto podría ser el caso de áreas temáticas olvidadas, poco desarrolladas o en grupos de población clásicamente desfavorecidos (p.ej., enfermedades raras, algunas enfermedades infecciosas y parasitarias, población pediátrica, etc.).

La integración mundial o globalización que viene acelerándose en las últimas décadas es otro aspecto a considerar ya que, lógicamente, también atañe a la investigación sanitaria. A medida que aumenta la integración se acelera el progreso tecnológico. De hecho, la investigación y el desarrollo de intervenciones sanitarias innovadoras llevada a cabo por países con políticas científicas y tecnológicas estables está permitiendo mejorar la salud y el bienestar de poblaciones de otros países.

Por último, a la vista de las limitaciones de tipo metodológico y práctico que puedan presentarse en este trabajo, parece necesario insistir en la conveniencia de seguir la línea de trabajo iniciada, potenciando la colaboración y el consenso de todos los agentes implicados. Resulta difícil establecer prioridades en base a un único criterio dada la complejidad de las diversas cuestiones ya comentadas. Los estudios de carga de enfermedad nos permiten presentar la dimensión de los problemas de salud de una población, aportan conocimiento sobre la importancia relativa y absoluta de las enfermedades y, por tanto, contribuyen al esta- 
blecimiento de prioridades. Cabe recordar que existen otros criterios (p.ej., calidad de las propuestas, relevancia de los proyectos, valor trasnacional y de transferencia a la práctica clínica, estructura y nivel del equipo investigador, oportunidades científicas, etc.) que también deberán ser tenidos en cuenta por los planificadores, gestores y evaluadores en su toma de decisiones.

\section{AGRADECIMIENTOS}

Al Fondo de Investigación Sanitaria del Instituto de Salud Carlos III y, concretamente, a su director y a las personas que con él trabajan en la gestión de las diferentes convocatorias, por facilitarnos la información de los proyectos y/o estudios financiados que han sido utilizados en el presente trabajo.

\section{BIBLIOGRAFÍA}

1. Ley $14 / 2007$, de 3 de julio, de Investigación Biomédica. Boletín Oficial del Estado núm. 159 de 4 de julio de 2007.

2. Plan Nacional de Investigación Científica, Desarrollo e Innovación Tecnológica 2004-2007. Madrid: Ministerio de Educación y Ciencia; 2003 [consultado, 15 de Junio 2008]. http://www.micinn.es/ciencia/plan_idi/

3. Plan Nacional de Investigación Científica, Desarrollo e Innovación Tecnológica 2008-2011. Madrid: Ministerio de Educación y Ciencia; 2007 [consultado, 15 de Junio 2008]. Disponible en: http://www.micinn.es/files/plan-nacional-consejo.pdf

4. Dixon J, Welch HG. Priority setting: lessons from Oregon. Lancet 1991;337(8746):891-4.

5. Ham C. Priority setting in health care: learning from international experience. Health Policy 1997;42(1):49-66.

6. Schopper D, Torres AM, Pereira J, Ammon C, Cuende N, Alonso M, Baylin A, Ronchi A, Rougemont A. Setting health priorities in a Swiss canton: what do different methods tell us? J Epidemiol Community Health 2000;54(5):388-93.
7. Pereira J, Cañón J, Álvarez E, Gènova R. La medida de los problemas de salud en el ámbito internacional: los estudios de carga de enfermedad. Rev Admin Sanitaria 2001;V(19):441-66.

8. Murray CJL, López A. The Global Burden of Disease. Boston: Harvard University Press; 1996.

9. Kim SY, Salomon JA, Goldie SJ. Economic evaluation of hepatitis B vaccination in low-income countries: using cost-effectiveness affordability curves. Bull World Health Organ 2007; 85(11): 833-42.

10. Navas E, Salleras L, Gisbert R, Domínguez A, Timoner E, Ibáñez D, Prat A. Cost-benefit and cost-effectiveness of the incorporation of the pneumococcal 7-valent conjugated vaccine in the routine vaccination schedule of Catalonia (Spain). Vaccine 2005;23(17-18):2342-8.

11. Chisholm D, van Ommeren M, Ayuso-Mateos JL, Saxena S. Cost-effectiveness of clinical interventions for reducing the global burden of bipolar disorder. Br J Psychiatry 2005;187:559-67.

12. Murray CJ, Lauer JA, Hutubessy RC, Niessen L, Tomijima N, Rodgers A, Lawes CM, Evans DB. Effectiveness and costs of interventions to lower systolic blood pressure and cholesterol: a global and regional analysis on reduction of cardiovascular-disease risk. Lancet 2003; 361(9359): 717-25.

13. Stuckler D, King L, Robinson H, McKee. WHO's budgetary allocations and burden of disease: a comparative analysis. Lancet 2008;372:1563-69.

14. Instituto Nacional de Estadística (INE). Fichero de microdatos anonimizado de defunciones con causa de muerte para el año 2006. Madrid: INE; 2008.

15. Coale A, Guo G. Revised regional model life tables at very low levels of mortality. Population Index 1989;55:613-43.

16. Gross CP, Anderson GF, Powe NR. The relation between funding by the National Institutes of Health and the burden-of-disease. N Engl J Med 1999;340(24):1881-7.

17. Curry CW, De AK, Ikeda RM, Thacker SB. Health burden and funding at the Centers for Disease Control and Prevention. Am J Prev Med 2006;30(3):269-76.

18. Lamarre-Cliché M, Castilloux A-M, LeLorier J. Association between the burden of disease and research funding by the Medical Research Coun- 
cil of Canada and the National Institutes of Health. A cross-sectional study. Clin Invest Med 2001;24:83-9.

19. Aoun S, Pennebaker D, Pascal R. To what extent is health and medical research funding associated with the burden of disease in Australia? Aust N Z J Public Health 2004;28:80-6.

20. Burnet NG, Jefferies SJ, Benson RJ, Hunt DP, Treasure FP. Years of life lost (YLL) from cancer is an important measure of population burden- and should be considered when allocating research funds. Br J Cancer 2005;92(2):241-5.

21. Report of the UK Health Research Analysis. Londres: UK Clinical Research Collaboration; 2006 [consultado, 26 de Julio 2008]. Disponible en: http://www.ukcrc.org/.

22. Schopper C, Pereira J, Torres A, Cuende N, Alonso M, Baylin A, Ammon C, Rougemont A. Estimating the burden of disease in one Swiss Canton: what do disability adjusted life years (DALY) tell us? Int J Epidemiol 2000;29:871-77.

23. Kominski GF, Simon PA, Ho A, Luck J, Lim YW, Fielding JE. Assessing the burden of disease and injury in Los Angeles County using disabilityadjusted life years. Public Health Rep 2002;117(2):185-91.

24. McKenna MT, Michaud CM, Murray CJL, Marks JS. Assessing the burden of disease in the United States using disability-adjusted life years. Am J Prev Med 2005;28(5):415-23.

25. Gènova R, Álvarez E, Morant C. Carga de enfermedad y tendencias de morbilidad de la población española. En: Puyol R y Abellán A (coords). Envejecimiento y dependencia. Una mirada al panorama futuro de la población española. Madrid: Mondiale Assistance 2006;6:107-24.

26. World Health Organization (WHO). The World Health Report 2007. A safer future : global public health security in the 21 st century. Geneva: World Health Organisation [consultado, 15 de Julio 2008]. Disponible en: http://www.who.int/whr/2007/ en/index.html

27. Instituto Nacional de Estadística (INE). Cifras de Población a 1 de julio de 2006. Estimaciones de la población actual. Madrid: INE; 2008. Disponible en: http://www.ine.es/

28. World Health Organisation (WHO). Revised Global Burden of Disease (GBD) 2002 Estimates. Incidence, prevalence, mortality, YLL, YLD and DALYs by sex, cause and region, estimates for 2002 as reported in the World Health Report 2004. Geneva: World Health Organisation [consultado, 10 de Julio 2008]. Disponible en: http://www.who.int/healthinfo/bodgbd2002revised/en/index.html

29. GesMor. Programa informático desarrollado por el Departamento de Salud Internacional, Instituto de Salud Carlos III. Con el apoyo de la Fundación Mexicana para la Salud (FUNSALUD) y el Banco Interamericano de Desarrollo, 2001. Disponible en: http://www.funsalud.org.mx/red-jlb/gesmor.htm

30. Orden SCO/1218/2004, de 15 de abril, por la que se establecen las bases reguladoras de la concesión de ayudas del Programa de Promoción de la Investigación Biomédica y en Ciencias de la Salud del Ministerio de Sanidad y Consumo, para la realización de proyectos de investigación en el marco del Plan Nacional de I+D+I 2004-2007. Boletín Oficial del Estado núm. 110 de 6 de mayo de 2004.

31. Orden SCO/1604/2005, de 31 de mayo, por la que se modifica la Orden SCO/1218/2004, de 15 de abril, por la que se establecen las bases reguladoras de la concesión de ayudas del Programa de Promoción de la Investigación Biomédica y en Ciencias de la Salud del Ministerio de Sanidad y Consumo, para la realización de proyectos de investigación en la marco del Plan Nacional de I+D+I 2004-2007. Boletín Oficial del Estado núm. 131 de 2 de junio de 2005.

32. Resolución de 7 de febrero 2006, del Instituto de Salud Carlos III, por la que se convocan ayudas del Programa de Promoción de la Investigación Biomédica y en Ciencias de la Salud del Ministerio de Sanidad y Consumo, para la realización de proyectos de investigación en el marco del Plan Nacional de I+D+I 2004-2007, durante el año 2006. Boletín Oficial del Estado núm. 47 de 24 de febrero de 2006.

33. Resolución de 10 de noviembre de 2006, del Instituto de Salud Carlos III, por la que se convocan ayudas del Programa de Promoción de la Investigación Biomédica y en Ciencias de la Salud del Ministerio de Sanidad y Consumo, para la realización de proyectos de investigación en el marco del Plan Nacional de I+D+I 2004-2007, durante el año 2007. Boletín Oficial del Estado núm. 272 de 14 de noviembre de 2006.

34. Resolución de 31 de marzo de 2006, del Instituto de Salud Carlos III, por la que se convocan ayudas del Programa de Promoción de la Investigación Biomédica y en Ciencias de la Salud, para la realización de estudios de investigaciones sobre evaluación de tecnologías sanitarias e investigación 
en servicios de salud en el marco del Plan Nacional I+D +I 2004-2007, durante el año 2006. Boletín Oficial del Estado núm. 98 de 25 de abril de 2006.

35. Resolución de 22 de marzo de 2007, del Instituto de Salud Carlos III, por la que se convocan ayudas del Programa de Promoción de la Investigación Biomédica y en Ciencias de la Salud para la realización de estudios de investigaciones sobre evaluación de tecnologías sanitarias e investigación en servicios de salud en el marco del Plan Nacional I+D+I 2004-2007, durante el año 2007. Boletín Oficial del Estado núm. 80 de 3 de abril de 2007.

36. Resolución de 16 de enero de 2007, del Instituto de Salud Carlos III, por la que se convocan ayudas del Programa de Promoción de la Investigación Biomédica y en Ciencias de la Salud para la realización de proyectos de investigación clínica de carácter no comercial con medicamentos de uso humano, en el marco de Plan Nacional de I+D+I 2004-2007, durante el año 2007. Boletín Oficial del Estado núm. 28 de 1 de febrero de 2007.

37. Plan de Calidad para el Sistema Nacional de Salud. Madrid: Ministerio de Sanidad y Consumo; 2007 [consultado, 26 de Diciembre 2007]. Disponible en: http://www.msc.es/organizacion/sns/planCalidadSNS/docs/planCalidad2007.pdf

38. Lomas J, Fulop N, Gagnon D, Allen P. On being a good listener: setting priorities for applied health services research. Milbank Q 2003;81(3):363-88.

39. Bernal-Delgado E, Peiró S, Sotoca R. Prioridades de investigación en servicios sanitarios en el Sistema Nacional de Salud. Una aproximación por consenso de expertos. Gac Sanit 2006;20(4):287-94.

40. Sánchez Martínez FI, Abellán-Perpiñán JM, Martínez Pérez JE. ¿Cómo se deben establecer y eva- luar las prioridades en salud y servicios de salud? Métodos de priorización y disparidades regionales. Informe SESPAS 2008. Gac Sanit 2008;22 (Supl 1): 126-36.

41. García-Altés A, Pinilla J, Peiró S. Aproximación a los pesos de calidad de vida de los «años de vida ajustados por calidad» mediante el estado de salud autopercibido. Gac Sanit 2006;20(6):457-64.

42. Cutler DM, Rosen AB, Vijan S. The value of medical spending in the United States, 1960-2000. N Engl J Med 2006;355:920-7.

43. Anand S, Hanson K. Disability-adjusted life years: a critical review. J Health Econ 1997;16(6): 685-702.

44. Murray CJL, Acharya AK. Understanding DALYs (disability-adjusted life years). J Health Econ 1997;16(6):703-30.

45. Williams A. Calculating the global burden of disease: time for a strategic reappraisal? Health Econ 1999;8:1-8.

46. Murray CJL, López AD. Progress and directions in refining the global burden of disease approach: a response to Williams. Health Econ 2000;9:6982.

47. Williams A. Comments on the response by Murray and López. Health Econ 2000;9:83-6.

48. Mathers CD, López AD, Stein C et al. "Deaths and Disease Burden by Cause: Global Burden of Disease Estimates for 2001 by World Bank Country Groups." Working Papers Series 18, Second Project on Disease Control Priorities in Developing Countries, World Health Organization; World Bank; and Fogarty International Center, U.S. National Institutes of Health,Washington, DC; 2004. 
